\title{
UPDATE
}

\section{Update in Geriatric Medicine}

\author{
Hollis D. Day, $M D, M S^{7}$, Elizabeth Eckstrom, $M D, M P H^{2}$, and Gail M. Sullivan, $M D, M P H^{3}$
}

'Division of General Internal Medicine, University of Pittsburgh School of Medicine, Pittsburgh, PA, USA; ${ }^{2}$ Division of General Internal Medicine and Geriatrics, Oregon Health and Science University, Portland, OR, USA; ${ }^{3}$ Division of Geriatric Medicine, University of Connecticut School of Medicine, Farmington, CT, USA.

KEY WORDS: geriatrics; dementia screening; gait speed; hip arthritis; osteoporosis; constipation.

$\mathrm{J}$ Gen Intern Med 24(3):421-6

DOI: $10.1007 / \mathrm{s} 11606-009-0909-9$

(c) Society of General Internal Medicine 2009

$\mathrm{O}$ lder adults constitute the largest subgroup in many general internal medicine practices, and providing optimal care to this population can be challenging. Common geriatric conditions, such as dementia, mobility disorders, and constipation, do not have well-evidenced detection strategies or treatments; fortunately, research in these areas is growing rapidly. Problems frequently encountered include detecting dementia, rapid office-based performance assessment, treating refractory osteoarthritic hip pain, treating osteoporosis in patients who cannot tolerate bisphosphonates, and treating constipation. We present this update in geriatric medicine to highlight recent key articles that have the potential to immediately improve or affect office practice by general internists.

We systematically identified articles by (1) reviewing ACP Journal Club and British Medical Journal's Bmjupdate for articles with high clinical relevance ratings for both general medicine and geriatric medicine (i.e., 6 out of 7 rating for ACP and 11 or higher rating for BMJ) and (2) ongoing review of major geriatrics/general medicine journals (New England Journal of Medicine, Annals of Internal Medicine, Journal of the American Medical Society, British Medical Journal, Journal of General Internal Medicine, Journal of the American Geriatrics Society, and Gerontologist). The time period chosen was March 2007 through March 2008. Reviewed articles were limited to the English language. Forty articles were identified; we then excluded those focused solely on settings (e.g., nursing home) and conditions (e.g., fear of falling) encountered less often in generalist practices. Finally, six articles were selected by consensus agreement based on their potential to immediately improve the care of older patients by generalists.

This review was presented at the 31st Annual SGIM Meeting in April 2008. There was no funding for this project.

Received July 31, 2008

Revised November 19, 2008

Accepted December 4, 2008

Published online January 29, 2009

\section{SCREENING}

\section{Screening for Dementia}

Systematic Review of the Optimum Office-based Strategies for Different Patient Subgroups

Holsinger T, Deveau J, Boustani M et al. Does this patient have dementia? JAMA. 2007;297:2391-2404.

The diagnosis of dementia in the outpatient setting is challenging; it is unclear which screening instruments are most valid and reliable in particular settings and patient groups, and under busy office time constraints. The authors of this study updated a previous systematic review by searching Medline and psychInfo from January 2000 through April 2006 using the terms exp Alzheimer's disease and exp dementia, sensitivity and specificity. Inclusion criteria for the studies included in this review were age over 60 and diagnosed with dementia using the criteria from the Diagnostic and Statistical Manual of Mental Disorders (i.e., dementia defined as disturbance in memory and at least one of the following: aphasia, apraxia, agnosia, and impaired executive functioning). Patients had to have a minimum of six years of education. Exclusion criteria included nonEnglish manuscripts; studies in inpatient or nursing home settings, or a memory disorder clinic without a control population; and studies using diagnostic imaging, laboratory, or physiological tests. From the eligible studies two independent reviewers assigned a quality rating based on the methods of the United States Preventive Services Task Force; only good quality studies performed in a community/primary care setting were utilized for the review. From these studies, 25 different screening instruments met criteria and were included. Results were reported in likelihood ratios (LR) positive (+) and negative (-) with $95 \%$ confidence intervals reported in parentheses when given.

The authors reported their results in three groups: the MiniMental State Examination (MMSE), brief instruments, and instruments for special situations.

The MMSE is a 30 -item survey testing orientation, language, attention, and visuo-spatial skills administered by a health professional with an average administration time of 7 min. The LR (+) was $6.3(3.4-47)$ and LR (-) was $0.19(0.06-$ 0.37) using a cutpoint of 24. Thus, the MMSE was a reasonable screening tool, but does have drawbacks, such as requiring an adjustment of the norms for age and education level. Additionally, the tool is copyrighted so that it can only be used if reproduced from memory, an original paper, or a source that is authorized by Psychological Assessment Resources.

Briefer instruments that were reviewed included an informant report of a patient's memory loss with a LR (+) of 6.5 (4.4- 
9.6). Also reviewed was the Memory Impairment Screen, in which subjects are given the names of items in four different categories (animals, vegetables, musical instruments, and cities) and after a brief delay asked to name the items in each category. This is a test of free recall ability, takes $4 \mathrm{~min}$ to perform, and had a LR (+) of 33 (15-72) and LR (-) of 0.08 (0.02-0.3). Finally, the authors reviewed the clock draw for which the LR (+) ranged from 1.2-7.7 and LR (-) from 0.130.71 . The clock draw is a rapid test and does not have to be adjusted for level of education. However, the test is dependent on the method of scoring used for example, an 8-point scale based on having a freely drawn circle with two hands of correct length at the correct time, versus a 15-point scale with similar characteristics but also including spacing of the numbers, and a variation of scoring in between.

Reviewed instruments for special situations included tests for patients with a high level of education. In the Hopkins Verbal Learning Test patients are given 12 words that they are then asked to recall in three separate trials. They are then presented with a word set that includes both the 12 original words and other "decoy" words to test recognition. This particular test had reasonable likelihood ratios [LR (+) of 4.849, LR (-) of 0.05-0.21]. The test is useful in highly educated patients, but does require purchase of the materials (approximately \$299 for a starter kit that includes the test manual and 25 test booklets) and requires some training in how to perform and score the testing.

While no test is perfect in all settings or all patients, the authors concluded that several valid instruments exist that will fit a busy practitioner's needs. The authors recommended using the MMSE to find cognitive impairment of at least moderate severity; the Hopkins verbal learning test or the word acquisition test if there is a suspicion of mild cognitive impairment or in a highly educated patient; and the Memory Impairment Screen or the Clock Drawing Test when there is minimal time available for screening (Table 1).

Limitations of the study included (1) using only English language studies; (2) some of the instruments discussed had only two studies to generate likelihood ratios, which suggests that they are not as well studied in primary care practices; and (3) studies were not usually designed with the primary endpoint of evaluating the performance of the screening instrument such that multiple biases were potentially introduced, all biasing toward improved performance of the specific instrument.

Screening for dementia is an important part of planning for a patient's future and determining adherence to a medical regimen. Health-care providers should be familiar with and have ready access to these screening instrument options for office practice.

This study was supported by the Institute for Medical Research at the Durham VA Medical Center, Paul B. Beeson K23 Career Development Award.

\section{Validated Brief Screen for the Detection of Early Dementia}

Grober, E, Hall C, Lipton RB, et al. Primary care screen for early dementia. J Am Geriatr Soc. 2008; 56:206-213.

Early detection of dementia allows patients to participate in future decision-making at a time when their cognitive function is still relatively intact; early detection may also avoid excess disability, such as from medication misuse, and enhance patient doctor communications. Internists need a rapid screening instrument. Detection of early dementia is likely to be complicated by a patient's educational level and ethnicity. This study sought to determine: (1) Is the Alzheimer's Disease Screen for Primary Care (ADS-PC) more sensitive to early dementia than the Mini Mental State Exam (MMSE). (2) Does the ADS-PC have as high a misclassification rate in minority patients or patients with limited education as the MMSE? The ADS-PC is a two-staged test that assesses free and prompted recall and verbal fluency, and can be administered by office personnel.

This was an observational, cross-sectional study of 316 African American and Caucasian patients including 55 patients with known early dementia. All patients were 65 years or older, had a family member/friend who had known them for at least 5 years, spoke English since age 30, and had adequate vision and hearing to complete testing. The study was performed in an urban geriatric clinic setting. Patients underwent not only the ADS-PC and MMSE, but also a complete neuropsychological battery of tests. Scores were reviewed and consensus regarding the presence or absence of early dementia, defined by DSM-IV criteria, was reached by a panel consisting of a neuropsychologist, geriatrician, and geriatric psychiatrist. Raters making the diagnosis of dementia were blinded to the results of the MMSE or the ADS-PC. The authors generated receiver operating curves for both the MMSE and the ADS-PC to visualize the differences in sensitivity and specificity for the full range of cutscores. The range of MMSE was then adjusted to match the same level of either specificity or sensitivity as the ADS-PC. In an analysis comparing sensitivities and using a cutoff score of 23 for the MMSE, the sensitivity for detection of early dementia was 0.75 for the ADS-PC and 0.53 for the MMSE. When comparing specificities, with a MMSE cutoff score of 26 the specificity for the ADS-PC was 0.9, and the MMSE was 0.73. Comparing odds ratios, the MMSE misclassified more than five times as many non-cases of dementia as the ADS-PC. Analyses by race demonstrated a sensitivity of 0.81 for the ADS-PC in African Americans and 0.39 for the MMSE. Analyses by level of education demonstrated a higher specificity (0.89 vs. 0.42) for the ADS-PC than the MMSE in patients with more than 12 years of education. However, there was no significant

Table 1. Summary of Screening Instruments for Dementia

\begin{tabular}{|c|c|c|c|}
\hline Instrument & Comments & LR (+) $(95 \% \mathrm{Cl})$ & LR (-) $(95 \% \mathrm{Cl})$ \\
\hline MMSE & $\begin{array}{l}\text { Familiar, takes about } 7 \text { min to perform, requires normative } \\
\text { adjustment for age and education level, English as primary } \\
\text { language, copyrighted }\end{array}$ & $6.3(3.4-47)$ & $0.19(0.06-0.37)$ \\
\hline Memory Impairment Screen & Brief, tests free recall, copyrighted & $33(15-72)$ & $0.08(0.02-0.3)$ \\
\hline Clock draw & $\begin{array}{l}\text { Brief, not dependent on education level/ability to speak English, } \\
\text { multiple scoring systems }\end{array}$ & $1.2-7.7$ & $0.13-0.71$ \\
\hline Hopkins Verbal Learning Test & Good for patients with higher education levels & $4.8-49$ & $0.05-0.21$ \\
\hline
\end{tabular}


difference in sensitivities in patients with more than 12 years of education and no differences in either sensitivities or specificities in patients with less than 12 years of education.

The authors concluded that the ADS-PC can be used in both African-Americans and Caucasians and has better specificity than the MMSE in those with more than 12 years of education, a population in which it is particularly challenging to diagnose early dementia. The limitations of the study include that it was a relatively small population and the testing was performed only on English-speaking African Americans and Caucasians and therefore may not be as applicable to other populations. In addition, the test characteristics of both screening instruments are likely to be less favorable when used prospectively in other populations, such as Latinos or other groups in whom English is not the primary language, as well as populations who may have mild cognitive impairment, rather than in this study where all patients were identified as having no dementia versus early dementia.

Screening for dementia is important for future planning, communicating patient wishes at a time when patients are still able to do so, and assisting practitioners in deciding the feasibility of treatment plans for other co-morbidities. The ADS-PC can be easily performed by office staff and may offer a practical strategy to identify patients with early cognitive impairment. This study was supported by the National Institute on Aging.

\section{Screening for Mobility Problems}

Hardy SE, Perera S, Roumani YF et al. Improvement in usual gait speed predicts better survival in older adults. J Amer Geriatr Soc 2007;55:1727-34.

As people age, mobility problems become more prevalent. Older patients may not complain to their doctors about changes in their gait or even falls. Studies of physical performance in older persons have shown associations between physical performance and key outcomes such as future function, falls, nursing home admission, and mortality. ${ }^{1-4}$ Research has also demonstrated that physical performance in older persons may be improved with interventions and that this improvement is associated with beneficial outcomes. However, improved gait speed, as a predictor of future outcomes, has been examined in only a few settings. ${ }^{5-7}$ This study examined the impact of 1-year improvement in physical performance, health status, or function on 8-year survival in older persons.

This prospective cohort study recruited 439 communitydwelling patients 65 years and older during 6 months in 1996 from two primary care clinics, a Medicare HMO and a VA. ${ }^{8}$ Inclusion criteria were: Mini-Mental State Examination of at least 16 , able to walk $4 \mathrm{~m}$, and gait speed between $0.2-1.3 \mathrm{~m} /$ s. Patients who used assistive devices were included. The primary outcome measure was mortality, as ascertained from the National Death Index through January 2007. Data were collected during home visits at baseline and 3, 6, 9, and 12 months on six measures: for physical performance $-4 \mathrm{~m}$ usual-pace gait speed and 12-point Short Physical Performance Battery; for health status - Eurobol and global health item from the Medical Outcomes Study 36-item Short Form Health Survey (SF-36); and for function - 16-item basic and instrumental activity of daily living scale from the National Health Interview Survey and the 100-point physical function index of the SF-36. A consensus panel defined criteria, based on literature review and clinical experience, for meaningful change in these six measures. Participants were assessed as improved, transiently improved, or never improved for each measure using the a priori definitions of meaningful change over 1 year.

Of the six measures, only improved gait speed (usual walking pace over $4 \mathrm{~m}$; improvement of $0.1 \mathrm{~m} / \mathrm{s}$ or better) was associated with survival at 8 years. Mortality at 8 years was $31.6 \%, 41.2 \%$, and $49 \%$ for those with improved, transiently improved, and never improved gait speed, respectively $(\mathrm{p}<0.001)$. The survival benefit for improvement at 1 year persisted after adjustment for covariates-demographics, comorbidity, cognitive function, and hospitalization (hazard ratio $0.42,95 \%$ CI $0.29-0.61, \mathrm{P}<0.001)$-and was consistent across subgroups based on age, sex, ethnicity, initial gait speed, health-care system, and hospitalization.

The authors concluded that gait speed over $4 \mathrm{~m}$, which can be readily measured without additional equipment in 2-3 min, may be an additional "vital sign" for older persons.

Older persons can be categorized as slow, intermediate, or fast walkers using cutpoints of 0.6 and $1.0 \mathrm{~m} / \mathrm{s} .{ }^{8}$ As an observational study, the linkage between improved gait speed and survival is unclear and may not be direct. Little clinical information was available to explain the relationship between gait speed and survival. Specific illnesses, such as cancer or hip fracture, may have affected both gait and mortality adversely in the performance of this test. However, internists should consider adding this simple measure, as a prognostic marker, to their routine assessment of older persons.

This study was sponsored by: Merck Research Laboratories, Pittsburgh Claude D. Pepper Center, National Institute on Aging, and Hartford Foundation Pittsburgh Center of Excellence in Geriatric Medicine. Merck employees participated in the design of the original study and the analyses to identify criteria for meaningful change. Merck employees also reviewed the manuscript and provided feedback.

\section{THERAPY \\ New Treatments for Osteoarthritis and Osteoporosis}

Lambert RGW, Hutchings EJ, Grace MGA et al. Steroid injection for osteoarthritis of the hip. A randomized, double-blind, placebo-controlled trial. Arthr Rheum 2007;56:2278-87.

The prevalence of hip osteoarthritis (OA) increases exponentially with age. Hip OA is painful, interferes with daily activities, and may result in poor sleep. Older patients may not tolerate non-steroidal anti-inflammatory medications (NSAID) due to side effects. Symptoms can affect function years before a hip replacement is necessary; in addition, some patients are not surgical candidates. Three randomized controlled trials of hip OA steroid injection were previously reported with mixed results, but the methods were flawed. ${ }^{9-11}$ Examples of problems seen in earlier studies include: used too large an injection volume, which made extravasation likely; measured only a dichotomous variable for outcome; and manipulated the hip joint on the day of injection and next 2 days rather than immobilization. 
As compared with knee injection, hip injection is technically difficult, and use of fluoroscopy is necessary to ensure that steroid is indeed injected into the synovial space. This study was carefully designed to definitively answer whether corticosteroid injection of the osteoarthritic hip improves pain and function and is safe.

The study was a randomized, double-blinded, placebocontrolled trial, with intention-to-treat analysis. The study inclusion criteria were: (1) primary hip OA; (2) age >40 years; (3) symptomatic disease for $\geq 6$ months; (4) persistent pain despite receiving maximum tolerated doses of acetaminophen (4 gm/d), and/or NSAID, with persistent pain defined as a minimum mean score of $40 \mathrm{~mm}$ on the five visual analog scales (VAS) for pain (0-100 $\mathrm{mm}$ for each) of the Western Ontario and McMaster Universities OA index (WOMAC) ${ }^{12}$, referred to as the WOMAC composite pain scale; (5) daily pain over prior month; (6) stable NSAID dose for 2 weeks; (7) can attend follow-up visits. Patients with diabetes mellitus, previous hip injection, anti-coagulant therapy or coagulopathy, allergy to the injected agents, and systemic arthritis were excluded. Patients were outpatients referred primarily by rheumatologists and orthopedic surgeons from the Capitol Health Region of Alberta, Canada. Most had severe disease and were awaiting hip replacement.

Patients were randomized to receive placebo (bipuvicaine and saline) or corticosteroid (bipuvicaine and triamcinolone hexacetonide) injected under fluoroscopy into the hip joint. Patients were instructed to rest for 3 days and undergo no exercise for another week, if possible. The primary outcome was pain improvement at 2 months [mean pain score (VAS) and $\geq 20 \%$ decrease in WOMAC composite pain score $\left(\mathrm{WOMAC}_{20}\right)$ ]. Secondary outcomes included $50 \%$ decrease in WOMAC composite pain score $\left(\mathrm{WOMAC}_{50}\right)$ at 2 months, changes to other WOMAC subscales, patient's global assessment of health (100 mm VAS), and SF-36 quality of life indices. Outcomes were assessed at baseline and 1,2, 3, and 6 months.

The study was terminated after interim data analysis of the first 52 OA patients enrolled because the results were so favorable toward steroid injection. The average age of the placebo group was significantly younger than the steroid group $-57 \pm 11$ years vs. $66 \pm 11$ years-but there was no difference in time since diagnosis of OA. There were no other significant differences between groups, such as use of NSAIDs, OA severity, or gender (about 60\% women in both groups). The mean WOMAC pain score fell $49.2 \%$ (310.1 to $157.4 \mathrm{~mm}$ ) at 2 months in the steroid group vs. $2.5 \%$ (314.3 to $306.5 \mathrm{~mm}$ ) in the placebo group $(\mathrm{p}<0.0001)$ (see Table 2). For the primary outcome, the proportion of $\mathrm{WOMAC}_{20}$ responders at 2 months was significantly higher in the steroid vs. placebo groups $(76.7 \%$ vs. $23.8 \%, \mathrm{p}<0.004)$. In secondary outcomes, WOMAC $_{50}$ responders were higher in the steroid vs. placebo groups $(61.3 \%$ vs. $14.3 \%, \mathrm{p}=0.001)$. The responses continued at 3 months $(58.1 \%$ vs. $9.5 \%, p=0.004)$. Other areas that similarly showed important clinical and statistically significant improvements with the intervention were WOMAC stiffness and physical function scores $(\mathrm{p}<0.0001)$, patient's global health scores $(\mathrm{p}=0.005)$, and SF-36 physical scores $(\mathrm{p}=0.04)$. There was no correlation between disease severity and treatment response. No differences in adverse events were seen, and there were no withdrawals due to adverse events. About $50 \%$ of patients in each group reported at least one adverse
Table 2. Outcomes from Steroid vs. Placebo Injection in Hip OA

\begin{tabular}{|c|c|c|c|}
\hline Primary & Placebo & Steroid & $\mathbf{P}$ \\
\hline Baseline mean pain $(\mathrm{mm})^{\&}$ & 314 & 310 & NS \\
\hline 2 months mean pain $(\mathrm{mm})^{\&}$ & 301 & 157 & $<0.0001$ \\
\hline $\mathrm{WOMAC}_{20}$ responders* & $24 \%$ & $68 \%$ & $<0.004$ \\
\hline \multicolumn{4}{|l|}{ Secondary } \\
\hline WOMAC $_{50}$ responders ${ }^{\#}$ & $14 \%$ & $61 \%$ & 0.001 \\
\hline WOMAC stiffness & 127 & 76 & 0.0001 \\
\hline WOMAC physical function & 949 & 539 & $<0.0001$ \\
\hline Global health & 60 & 45 & 0.005 \\
\hline SF-36 physical function $\$$ & 23 & 33 & 0.008 \\
\hline
\end{tabular}

${ }^{\&}$ Western Ontario and McMaster Universities Osteoarthritis index (WOMAC) pain subscale score

${ }^{*}$ Persons with $\geq 20 \%$ decrease in WOMAC composite pain score at 2 months

\#Persons with $\geq 50 \%$ decrease in WOMAC composite pain score at 2 months

\$Short Form-36 physical function component score

event in the 2-month period; most of these were mild or considered unrelated to treatment. Of note, there were no infections.

Due to obvious symptomatic improvements, interim data analysis was recommended, showed highly significant differences between treatments, and led to early study termination. The authors concluded that hip steroid injection under fluoroscopy is highly effective for pain relief and improved function in symptomatic OA, with benefits lasting 3 months, and no adverse side effects up to 6 months. The study is limited by whether the results can be generalized to patients with less severe OA. Further studies should elucidate how often injections may be performed and what effect, if any, injections have on disease progression.

The results from this study should prompt clinicians to refer patients with hip OA that is symptomatic, unresponsive to maximum tolerated doses of analgesia and other medical treatments, and interferes with quality of life/function to interventional radiologists for corticosteroid injection. This study was sponsored by: CHAR/Nycomed Development Award, MSI Foundation, University of Alberta Hospital Foundation, and Arthritis Society of Canada.

\section{Zoledronic Acid for Osteoporosis Treatment}

Black, DM, Delmas, PD, Eastell, R, et al. Once-yearly zoledronic acid for treatment of postmenopausal osteoporosis. NEJM 2007;356:1809-22.

Osteoporosis is an important cause of disability in postmenopausal women, and costs of care for treatment of osteoporosis top $\$ 18$ billion dollars annually in the US. ${ }^{13}$ Oral bisphosphonates (e.g., alendronate, residronate) are effective in reducing fractures in post-menopausal women with osteoporosis, but studies show that as many as 50\% of women who begin oral bisphosphonates are no longer adherent after 1 year of therapy. ${ }^{14},{ }^{15}$ This study seeks to determine if a once-yearly infusion of zoledronic acid reduces fracture risk compared to placebo in post-menopausal women with osteoporosis, over a 3-year period.

The HORIZON Pivotal Fracture Trial, an international, multi-center study led by an investigator team at the University of California at San Francisco (UCSF), was a randomized, double-blind, placebo-controlled trial comparing a 15-min IV 
infusion of zoledronic acid ( $5 \mathrm{mg}$ ) or placebo at baseline (day 0), at 12 months, and at 24 months. Over 7,000 post-menopausal women (mean age 73 , range $65-89$ ) with a $T$ score of -2.5 or less at the femoral neck, with or without evidence of existing vertebral fracture or a $\mathrm{T}$ score of -1.5 or less with radiologic evidence of at least two mild vertebral fractures or one moderate vertebral fracture, entered the study. Previous use of oral bisphosphonates was allowed, with a washout period dependent on prior use (e.g., $>48$ month prior use required a 2-year washout). Other treatments-hormone replacement therapy, raloxifene, calcitonin, tibolone, tamoxifen, dihydroepiandrosterone, ipriflavone, and medoxyprogesterone-were allowed at baseline and during follow-up. Patients were stratified by use of non-bisphosphonate osteoporosis medications at baseline (Stratum 1: not taking any osteoporosis medications at baseline; Stratum 2: taking other osteoporosis medications at baseline). Exclusion criteria were prior use of parathyroid hormone, fluoride, anabolic steroids, growth hormone, and strontium, or a creatinine clearance less than 30 .

Zoledronic acid reduced radiographic vertebral fractures (defined as a reduction in vertebral height of at least $20 \%$ and $4 \mathrm{~mm}$ by quantitative morphometry) and hip fractures with a relative risk of 0.3 and 0.59 , respectively (Table 3). Bone mineral density increased significantly at the total hip, lumbar spine, and femoral neck when compared with the placebo group. Adverse events were common in both groups $195.5 \%$ in zoledronic acid group and $93.9 \%$ in placebo, NS). The most common post-dose adverse events in the zoledronic acid group were: $1.3 \%$ transient increase in creatinine (back to baseline by 30 days); chills, nausea, bone pain, and back pain; arrhythmia including atrial fibrillation (6.9\% in zoledronic acid group compared with $5.3 \%$ in placebo group); and a transient decrease in calcium level.

Zoledronic acid is effective in reducing fractures in postmenopausal women, with an efficacy similar to that of oral bisphosphonates. It has not been compared head to head with oral bisphosphonates. Though this was a large population, it was probably not sufficient to sort out true risks; therefore, continued adverse event surveillance is necessary. Once-yearly zoledronic acid improves compliance over oral bisphosphonates and is a reasonable choice for osteoporosis patients who do not tolerate or are non-compliant with oral bisphosphonates. Of note, zoledronic acid costs approximately $\$ 1,100$ for a once-yearly injection (not including infusion costs), comparing unfavorably with generic alendronate, and is not covered by many Medicare Part D plans. This study was sponsored by Novartis Pharma; many on the investigator team received support from Novartis. The study was jointly designed by members of the steering committee and the sponsor. The sponsor had responsibility for data collection and quality control. UCSF hosted the data and safety monitoring board. Analysis was performed by the sponsor, but independently

Table 3. Three-year Outcomes, Zoledronic Acid vs. Placebo

\begin{tabular}{|c|c|c|c|c|c|}
\hline & Placebo & $\begin{array}{l}\text { Zoledronic } \\
\text { acid }\end{array}$ & $\mathrm{RR}(\mathrm{Cl})$ & ARR & NNT \\
\hline $\begin{array}{l}\text { Vertebral } \\
\text { fracture }\end{array}$ & $10.9 \%$ & $3.3 \%$ & $0.3(0.24-0.38)$ & $7.6 \%$ & 13 \\
\hline Hip fracture & $2.5 \%$ & $1.4 \%$ & $0.59(0.42-0.83)$ & $1.1 \%$ & 91 \\
\hline Any fracture & $12.8 \%$ & $8.4 \%$ & $0.67(0.58-0.77)$ & $4.4 \%$ & 23 \\
\hline
\end{tabular}

Table 4. Polyethylene Glycol vs. Placebo for Constipation

\begin{tabular}{llll}
\hline \hline & $\begin{array}{l}\text { PEG } \\
(\mathbf{N}=204)\end{array}$ & $\begin{array}{l}\text { Placebo } \\
(\mathbf{N}=100)\end{array}$ & $P$ \\
\hline Primary definition, \% of weeks & $61.4 \%$ & $21.8 \%$ & $<0.001$ \\
ROME criteria not met, \% of weeks & $66.2 \%$ & $24.4 \%$ & $<0.001$ \\
\hline
\end{tabular}

confirmed by investigators at UCSF. The article was approved by the 13-member steering committee, which included two representatives from the sponsor.

\section{Polyethylene Glycol Laxative for Chronic Constipation}

DiPalma, JA, Cleveland, MvB, McGowan, J et al. A randomized, multicenter, placebo-controlled trial of polyethylene glycol laxative for chronic treatment of chronic constipation. Am J Gastroenterol 2007;102:1436-1441.

Affecting at least 63 million Americans, chronic constipation is a common complaint in primary care practice and is especially prevalent in patients over $65 .{ }^{16}$ Until recently, few data have been available about long-term treatment of chronic constipation. ${ }^{17}$ Tegaserod, a serotonin subtype 4 receptor partial agonist, was recently taken off the market, and lubiprostone, a selective chloride channel activator, has not been studied in patients with decreased renal function. The current study asks the question: is polyethylene glycol (PEG) safe and effective for extended ( 6 months) use in patients with chronic constipation?

Three hundred four subjects met an historical definition of constipation based on modified ROME criteria: on average, for the preceding 3 months, when not taking laxatives, satisfactory stool was less frequent than three per week, and one or more of the following were met: straining in more than $25 \%$ of defecations, lumpy or hard stools in more than $25 \%$ of defecations, and sensation of incomplete evacuation in more than $25 \%$ of defecations ${ }^{18}$. Subjects were randomized in a $2: 1$ ratio (PEG to placebo) to receive PEG or placebo. Seventy-five patients aged over 65 were included in the study. Subjects were instructed to mix the contents of one packet ( $17 \mathrm{~g}$ ) in $8 \mathrm{oz}$ of juice or other beverage and drink once daily. Subjects called an Interactive Voice Response System daily to report their bowel movement experiences for that day and answer questions related to study efficacy and safety criteria. The primary efficacy endpoint was assessed on the basis of a calculated

Table 5. Changes to Clinical Practice Emerging from Articles in the Past Year

\begin{tabular}{l}
\hline \hline Start \\
- Referring patients with symptomatic hip OA, unresponsive to \\
medical treatments, to interventional radiologists for steroid hip \\
injection \\
- Using polyethylene glycol for generally healthy patients with \\
refractory, chronic constipation \\
Consider \\
- Adding brief dementia screens to your office routine: Clock Draw \\
Test, Memory Impairment Screen, or ADS-PC \\
- Training your office staff to measure usual walking speed annually in \\
patients 65 years and older \\
- Using annual IV zoledronic acid for women with osteoporosis who are \\
unable to take oral bisphosphonates
\end{tabular}

- Referring patients with symptomatic hip OA, unresponsive to injection

- Using polyethylene glycol for generally healthy patients with refractory, chronic constipation

- Adding brief dementia screens to your office routine: Clock Draw Test, Memory Impairment Screen, or ADS-PC patients 65 years and olde

unable to take oral bisphosphonates 
success rate for the treatment period. Weekly treatment success was defined according to three elements: no use of rescue laxative, satisfactory stool greater than or equal to three per week, and one or fewer of the remaining ROME-based symptom criteria listed above.

Subjects treated with PEG had a 52\% success rate compared to $11 \%$ in the placebo group ( $\mathrm{p}<0.001$ ) (Table 4). Older patients achieved similar efficacy rates $(46 \%$ difference favoring PEG). Subjects in the PEG group reported more GI side effects from treatment, including abdominal distension, diarrhea, loose stools, flatulence, and nausea (PEG 39.7\%, placebo $25 \%, P=0.15)$. There were no clinically significant laboratory changes.

Chronic constipation is a common problem in adult and elderly patients. This study demonstrates that PEG is both safe and effective for continual use for up to 6 months in these populations. PEG had increased GI side effects compared to placebo, but no significant electrolyte abnormalities. Study limitations include the fact that the authors do not report whether data collection and analysis were blinded. Patients in the study were generally healthy, so extrapolation of results to ill or frail older adults should be done with caution. Of note, these patients had suffered from constipation for an average of 23 years, and PEG treatment resulted in a rapid increase in the number of successfully treated patients in the first month, with the maximum response by the second month, and the response remaining fairly level thereafter. If these results are replicated in usual clinical practice, PEG may become an important addition to our treatment strategies for chronic constipation. This study was sponsored by Braintree Laboratories, Inc. All authors are consultants to or employees of Braintree Laboratories.

Based on the above review of these recent articles, clinicians should consider adjusting their care of older patients. In Table 5 recommendations for actions to "start" and those to "consider" are listed.

Acknowledgements: This work was presented at the 31st Annual Meeting of the Society for General Internal Medicine. There was no funding provided for this work.

Conflict of Interest: None disclosed.

Corresponding Author: Hollis D. Day, MD, MS; Division of General Internal Medicine, University of Pittsburgh School of Medicine, M211 Scaife Hall 3550 Terrace Street, Pittsburgh, PA 15261-1211, USA (e-mail: dayh@upmc.edu).

\section{REFERENCES}

1. Lee $\mathbf{Y}$. The predictive value of self assessed general, physical, and mental health on functional decline and mortality in older adults. J Epidemiol Community Health. 2000;54:123-9.

2. Lee S, Lindquist $\mathbf{K}$, Segal $\mathbf{M}$, Covinsky $\mathbf{K}$. Development and validation of a prognostic index for 4-year mortality in older adults. JAMA. 2006;295:801-8.

3. Gurlanik J, Simonsick E, Ferruci L, et al. A short physical performance battery assessing lower extremity function: association with selfreported disability and prediction of mortality and nursing home admission. J Gerontol. 1994;49:M85-94.

4. Cesari M, Kritchevsky S, Penninx B, et al. Prognostic value of usual gait speed in well-functioning older people - results from the Health, Aging and Body Composition Study. J Am Geriatr Soc. 2005;53:1675-80.

5. Friedman PJ, RIchmond DE, Baskett JJ. A prospective trail of serial gait speed as a measure of rehabitliation in the elderly. Age Aging. 1998; 17:227-35.

6. Purser JL, Weinberger M, Cohen HJ, et al. Walking predicts health status and hospital costs for frail elderly male veterans. J Rehabil Res Dev. 2005;42:535-46.

7. Shah MR, Hasselblad V, Gheorghiade M, et al. Prognostic usefulness of the six-minute walk in patients with advance congestive heart failure secondary to ischemic or nonischemic cardiomyopathy. Am J Cardiol. 2001;88:987-93.

8. Studenski S, Perera S, Wallace $\mathbf{D}$, et al. Physical performance measures in the clinical setting. J Am Geriatr Soc. 2003;41:314-22.

9. Qvistgaard $\mathbf{E}$, Christensen $\mathbf{R}$, Torp-Pedersen $\mathbf{S}$, Liddal $\mathbf{H}$. Intraarticular treatment of hip osteoarthritis: a randomized trial of hyaluronic acid, corticosteroid, and isotonic saline. Osteoarthritis Cartilage. 2006; 14:163-79.

10. Kullenbery B, Runesson R, Tuvhag R, Olsson C, Resch S. Intraarticular corticosteroid injection: pain relief in osteoarthritis of the hip? J Rheumatol. 2004;31:2265-8.

11. Flanagan J, Casale F, Thomas T, Desai K. Intra-articular injection for pain relief in patients awaiting hip replacement. Ann R Coll Surg Engl. 1988;70:156-7.

12. Bellamy N, Buchanan W, Goldsmith C, Campbell J, Stitt L. Validation study of WOMAC: a health status instrument for measuring clinically important patient relevant outcomes to anti-rheumatic drug therapy in patients with osteoarthritis of the hip or knee. J Rheumatol. $1988 ; 15: 1833-40$.

13. Department of Health and Human Services. Bone Health and Osteoporosis: A Report of the Surgeon General. Rockville, MD: Office of the Surgeon General; 2004.

14. Cramer J, Amonkar M, Hebborn A, Altman R. Compliance and persistence with bisphosphonate dosing regimens among women with postmenopausal osteoporosis. Curr Med Res Opin. 2005;21:1453-60.

15. Lo J, Pressman A, Omar M, Ettinger B. Persistence with weekly alendronate therapy among postmenopausal women. Osteoporos Int. 2006; 17:922-8.

16. Higgins $\mathbf{P}$, Johanson J. Epidemiology of constipation in North America: a systematic review. Am J Gastroenterol. 2004;99:750-9.

17. Lembo A, Camilleri M. Chronic constipation. N Engl J Med. 2003;349:1360-8

18. Whitehead WE, Chaussade S, Corazziari E, et al. Report of an international workshop in management of constipation. Gastroenterol Int. 1991;4:99-113. 